\title{
SOME HARDY INEQUALITIES ON THE SPHERE
}

\section{YINGXIONG XIAO}

Abstract. In this paper we establish some Hardy inequalities related to the geodesic distance on the sphere and compute the corresponding sharp constants. Moreover, some Rellich inequalities have been also established and the constants are also sharp.

Mathematics subject classification (2010): 26D10, 46E36.

Keywords and phrases: Hardy inequality, Rellich inequality, sphere, sharp constant.

\section{REFERENCES}

[1] B. Abdellaoui, D. Colorado, I. Peral, Some improved Caffarelli-Kohn-Nirenberg inequalities, Calc. Var. Partial Differential Equations, 23, (2005), 327-345.

[2] Adimurthi, S. Filippas, A. Tertikas, On the best constant of Hardy-Sobolev inequalities, Nonlinear Analysis, 70, (2009), 2826-2833.

[3] G. Barbatis, S. FilipPas And A. Tertikas, A unified approach to improved $L^{p}$ Hardy inequalities with best constants, Trans. Amer. Math. Soc., 356, (2004), 2169-2196.

[4] P. BARAs, J. A. Goldstein, The heat equation with a singular potential, Trans. Amer. Math. Soc. 284, (1984), 121-139.

[5] H. BReZis, J. L. VÁZQueZ, Blow-up solutions of some nonlinear elliptic problems, Rev. Mat. Univ. Comp. Madrid, 10 (1997), 443-469.

[6] G. CARRon, Inégalités de Hardy sur les variétés riemanniennes non-compactes, J. Math. Pures Appl. 76, (1997), 883-891.

[7] L. D'Ambrosio, S. Dipierro, Hardy inequalities on Riemannian manifolds and applications, Annales de l'Institut Henri Poincare (C) Non Linear Analysis, 31, 3 (2014), 449-475.

[8] E. B. Davies, A. M. HinZ, Explicit constants for Rellich inequalities in $L_{p}(\Omega)$, Math. Z., 227 (1998), 511-523.

[9] S. Filippas, A. Tertikas, Optimizing improved Hardy inequalities, J. Funct. Anal. 192, 1 (2002), $186-233$.

[10] S. Gallot, D. Hulin, J. Lafontaine, Riemannian Geometry, 3rd edn. Springer-Verlag, Berlin, 2004.

[11] H. JABer, Optimal Hardy-Sobolev Inequalities on Compact Riemannain Manifolds, J. Math. Anal. Appl. 421 (2015), 1869-1888.

[12] I. Kombe, M. ÖZAYDIn, Improved Hardy and Rellich inequalities on Riemannian manifolds, Trans. Amer. Math. Soc. 361, (2009), 6191-6203.

[13] I. KomBe, M. ÖZAYDIN, Hardy-Poincaré, Rellich and Uncertainty principle inequalities on Riemannian manifolds, Trans. Amer. Math. Soc. 365 (2013), 5035-5050.

[14] J. Moser, A sharp form of an inequality by N. Trudinger, Indiana Univ. Math. J. 20 (1970), $1077-$ 1092.

[15] J. Moser, On a non-linear problem in differential geometry and dynamical systems, Academic Press, N.Y. (ed. M. Peixoto) 1973.

[16] Q. YANG, D. SU, Y. Kong, Hardy inequalities on Riemannian manifolds with negative curvature, comm. contemp. Math. 16, 1350043 (2014) [24 pages].

[17] J. L. VAZQUeZ, E. ZuAZUA, The Hardy inequality and the asymptotic behaviour of the heat equation with an inverse-square potential, J. Funct. Anal. 173 (2000), 103-153. 